\title{
CONTRIBUTO ALLO STUDIO DELLA SUFFISSAZIONE VERBALE NELL'ITALIANO CONTEMPORANEO Raffronto contrastivo
}

\section{INTRODUZIONE}

La formazione delle parole comprende tre parti fondamentali: la formazione delle parole mediante suffissi (la suffissazione), la composizione delle parole nonché la formazione delle parole mediante prefissi (la prefissazione). I risultati di questi tre procedimenti formativi sono rispettivamente il derivato o il suffissato, il composto o la parola composta e il prefissato (che può essere derivato o composto). La suffissazione comprende le formazioni tratte dalle diverse basi (nominali, aggettivali e verbali); la tripartizione è fatta prendendo in considerazione il punto di partenza del procedimento di formazione della parola, vuol dire considerando la base della nuova formazione: si hanno i suffissati denominali, deaggettivali e deverbali. Se si prende in considerazione il punto di arrivo del procedimento formativo si hanno ancora tre tipi di nuove formazioni; per quanto riguarda la categoria di arrivo si hanno i suffissati nominali, aggettivali e verbali.

Riportiamo queste nozioni introduttive che sono conosciute a linguisti e a tutti coloro che studiano la formazione delle parole e il lessico di una lingua. Dato che nell'ambito dello studio della formazione delle parole la terminologia differisce qualche volta da una scuola linguistica all'altra e presenta spesso delle difficoltà nel capire a fondo il lavoro che tratta tale materia, abbiamo preferito aggiungere queste spiegazioni terminologiche necessarie per facilitare la lettura di questo articolo.

1.1. In italiano la formazione dei verbi riguarda innanzi tutto la suffissazione o la formazione mediante suffissi e la prefissazione o la formazione mediante prefissi (i prefissi e i suffissi sono chiamati ancora, tutti e due, affissi). Inoltre nella lingua italiana viene usata frequentemente la formazione parasintetica dei verbi. Quindi la formazione dei verbi italiani comprende i seguenti procedimenti formativi: la suffissazione, la prefissazione e la formazione parasintetica. La composizione dei verbi è poco sviluppata.

1.2. Per quanto riguarda la formazione dei verbi si tratta infatti di una bipartizione: a) i suffissati o i verbi formati mediante suffissi e b) i prefissati o i verbi formati mediante prefissi. Ma a questa bipartizione va aggiunta la formazione parasintetica che è a cavallo tra suffissazione e prefissazione: il risultato di questo procedimento formativo è 
il parasintetico. La formazione parasintetica è frequentissima nella formazione dei verbi italiani da basi aggettivali e da quelle nominali; essa consiste nell'aggiungere un prefisso e un suffisso sullo stesso livello o simultaneamente e distingue la formazione dei verbi da tutte le altre formazioni (dalla formazione dei nomi e da quella degli aggettivi, dato che in esse $i$ parasintetici sono rari: incappucciato, l'incappucciato). In altri termini, soltanto la formazione verbale presenta questo modo caratteristico di formare le parole - i verbi parasintetici oppure i parasintetici, mentre la formazione nominale e quella aggettivale presentano di regola due procedimenti formativi separati: la suffissazione e la prefissazione.

1.3. La formazione simultanea - parasintetica, propria dei verbi, va studiata insieme con la suffissazione verbale, dato che gli stessi suffissi servono a formare i verbi parasintetici e i suffissati, e, in ambedue i casi il risultato del procedimento formativo è il verbo formato. Il parasintetico è a metà strada tra suffissazione e prefissazione.

1.3.1. I prefissi si distinguono dai suffissi in quanto precedono la base e soprattutto in quanto alcuni prefissi sono autonomi (fungono da preposizioni e da avverbi) e formano parole composte; per cui la formazione delle parole mediante prefissi appartiene alla derivazione e alla composizione. I verbi formati mediante suffissi sono dei derivati, mentre i verbi formati mediante prefissi possono essere dei derivati o dei composti; quindi si tratta di derivati o di composti parasintetici.

1.3.2. La formazione mediante suffissi ha la proprietà di transcategorizzare (nomi derivati tratti da aggettivi e da verbi, aggettivi derivati tratti da nomi e da verbi, verbi derivati tratti da nomi e da aggettivi). Nella suffissazione ci sono suffissati che non transcategorizzano (nomi derivati tratti da nomi, aggettivi derivati tratti da aggettivi, verbi derivati tratti da verbi).

La formazione mediante prefissi non transcategorizza, fatta eccezione per i parasintetici.

Nella formazione parasintetica si ha simultaneamente la prefissazione e la suffissazione. La formazione parasintetica transcategorizza, dunque la transcategorizzazione è ottenuta con il suffisso.

1.4. In questo articolo abbiamo esaminato insieme $i$ verbi derivati tratti da basi nominali e da quelle aggettivali; i verbi vanno trattati insieme se si considera la formazione dei verbi dal punto di vista contrastivo, comparando i meccanismi formativi delle due lingue che si confrontano. Riteniamo che il metodo contrastivo sia capace di costituire un quadro di riferimento per il problema che ci interessa in questo lavoro. La scelta del metodo contrastivo non comporta il rifiuto di altri metodi di ricerca, è soltanto una scelta operativa. Nella nostra analisi la lingua di partenza è il croato, mentre la lingua di arrivo è l'italiano, quindi dobbiamo cercare gli equivalenti italiani dei verbi croati: sono gli equivalenti semantici e formali dei verbi croati esaminati. Sono presi in considerazione solo i suffissi produttivi; i suffissi poco produttivi o non produttivi non si prendono in esame. 
I verbi derivati tratti da nomi e da aggettivi presentano molte caratteristiche comuni, soprattutto per quanto riguarda gli equivalenti formali e semantici nella lingua che si confronta. E' la ragione principale per cui vengono trattati insieme i verbi denominali e quelli deaggettivali.

\section{I VERBI DENOMINALI}

I verbi tratti da basi nominali e formati mediante suffissi presentano una grande diversità di significati, ma in linea di massima essi indicano l'azione in relazione al nome che è la base del verbo.

2.1. Il suffisso croato -ati forma verbi tratti da nomi indicanti vari oggetti. I verbi croati e i corrispondenti verbi italiani indicano per lo più l'azione fatta con il nome che è la base del verbo: batinati (da batina) - bastonare 'percuotere con un bastone'; blanjati (da blanja) - piallare 'lavorare, spianare e assottigliare con la pialla'; četkati (da četka) spazzolare 'pulire con la spazzola'; grabljati (grablje) - rastrellare 'radunare in mucchio col rastrello'; mamuzati (dal nome mamuza) - spronare 'stimolare il cavallo con gli sproni'; šibati (da šiba) - vergheggiare 'percuotere con verga'.

2.1.1. Il suffisso croato -ati forma frequentemente i verbi il cui significato viene espresso con un verbo transitivo e con il nome (base del derivato) in funzione di oggetto diretto: čipkati (da čipka) - merlettare 'applicare merletti'; listati (da list) - frondeggiare 'mettere fronde'; sedlati (da sedlo) - sellare 'mettere la sella'.

In questo gruppo possono rientrare pure i verbi skijati se (da skija) - sciare 'praticare lo sport dello sci' e veslati (da veslo) - remare 'maneggiare i remi'.

2.1.2. Se la base del verbo è un nome che indica strumento musicale, il verbo significa 'suonare questo strumento': bubnjati - tamburare 'battere il tamburo'; frulati pifferare 'suonare il piffero'.

Gli equivalenti semantici e formali dei verbi croati formati mediante il suffisso -ati, sono i verbi italiani derivati da basi nominali mediante suffissi (-are, -eggiare). Però molti verbi croati non hanno verbi equivalenti italiani, formati mediante suffissi, e il loro significato si può esprimere con i verbi parasintetici: klupčati - aggomitolare 'avvolgere in gomitoli', oppure con i costrutti sintattici costituiti dal verbo e dal nome (solo o accompagnato da un complemento) che traduce la base del verbo croato: grudati se (da gruda) 'tirare le palle di neve', kaljužati se (da kaljuža) 'voltarsi nel fango, detto di maiali', kuglati se (da kugla) 'giocare alle bocce, fare una partita alle bocce', orgijati (da orgija) 'far baldoria', sunčati se (da sunce) 'prendere un po' di sole, prendere un bagno di sole, mettersi (o stare) al sole'.

2.2. Il suffisso croato -iti forma verbi derivati da basi nominali. I verbi hanno significati molto diversi, ma tutti indicano l'azione in relazione al significato del nome che è la base del suffissato. I verbi si possono dividere, secondo il loro significato, in due grandi gruppi: il primo gruppo comprende i verbi derivati da nomi indicanti esseri 
viventi - esseri umani e animali, mentre il secondo gruppo comprende i verbi derivati da nomi indicanti oggetti concreti.

2.2.1. I suffissati con base nominale, indicanti esseri viventi - umani e animali, significano 'l'azione o l'attività propria degli esseri viventi'. Naturalmente l'ambito di questa attività è vasto e possiamo fare distinzioni ulteriori per quanto riguardla il significato di questi derivati i quali sviluppano significati particolari e differenziali.

2.2.1.1. Il suffisso croato -iti serve a formare verbi che significano 'esercitare il mestiere' indicato dal sostantivo che è la base del verbo: gospodariti (da gospodar) - padroneggiare 'comandare/controllare qcn. o qc. come un padrone'; krijumčariti (da krijumčar) - contrabbandare 'introdurre una merce di contrabbando'.

L'equivalente semantico e formale dei suffissati croati è il verbo italiano derivato, formato mediante un suffisso (-are, -eggiare). Però molti di questi verbi croati non hanno verbi equivalenti italiani, e il loro significato deve essere espresso mediante uno dei costrutti sintattici costituiti da un verbo (essere o fare) e dal nome che traduce la base del verbo croato: drvariti (da drva) 'far legna', mešetariti (da mešetar) 'fare il sensale, fare da mediatore', piljariti (da piljar) 'fare il rivendugliolo', stražariti (da stražar) 'essere di guardia, fare la sentinella'.

2.2.1.2. Il suffisso croato -iti serve a formare verbi che significano 'comportarsi come l'essere umano o animale' indicato dal nome che è la base del verbo croato: cjepidlačiti (dal nome cjepidlaka) - pedanteggiare 'fare il pedante'; kopuniti se (da kopun) - pavoneggiarsi.

Gli equivalenti formali e semantici dei verbi croati sono i derivati italiani con base nominale formati mediante il suffisso -eggiare. Alcuni verbi croati di questo sottogruppo semantico non hanno forme verbali equivalenti, ma $\mathrm{i}$ loro equivalenti sono $\mathrm{i}$ costrutti sintattici italiani che traducono il significato del verbo croato: kicošiti se (da kicoš) 'fare il bellimbusto, fare lo zerbinotto'; tvrdičiti (da tvrdica) 'fare lo spilorcio'.

2.2.1.3. I suffissati croati in -iti che significano 'far diventare' quello che indica il nome che è la base del verbo non hanno forme derivate equivalenti in italiano; $i$ loro equivalenti sono i costrutti sintattici italiani che sono costituiti dal verbo transitivo che significa 'ordinare, consacrare' e sim. e dal nome che traduce la base del verbo croato: đakoniti (da đakon) 'ordinare qcn. diacono'; popiti (da pop) 'ordinare qcn. prete'; vladičiti (da vladika nella chiesa cristiana ortodossa) 'consacrare qcn. vladika, vescono'.

Tutti e tre i verbi esaminati appartengono alla terminologia ecclesiastica.

2.2.1.4. Il suffisso croato -iti forma verbi che significano 'diventare quello che indica il nome' che è la base del verbo; questi suffissati croati non hanno forme equivalenti formate mediante suffissi in italiano. L'equivalente semantico del verbo può essere il costrutto sintattico italiano, costituito dal verbo divenire, diventare e sim. e dal sostantivo che traduce la base del verbo croato: popiti se (da pop) diventar prete'; vladičiti se (da vladika) 'diventar vescovo'. 
2.2.1.5. Il suffisso croato -iti serve a formare verbi che significano 'figliare detto di animali'. Il croato forma con facilità questi suffissati, mentre l'equivalente italiano è il verbo figliare usato da solo o accompagnato dal nome dell' animale giovane: la mucca ha figliato, la scrofa ha figliato i maialini. L'esemplificazione è numerosa, perché si vuole mettere in risalto la differenza nell' esprimere gli stessi concetti nelle due lingue: janjiti se (da janje) 'figliare detto di pecora'; koziti se (da koza) 'figliare detto di capra'; maciti se (dal nome maca) 'figliare detto di gatta', prasiti se (da prase) 'figliare detto di scrofa', šteniti se (da štene) 'figliare detto di cagna'; teliti se (detto di mucca), ždrijebiti se (detto di mucca).

Come risulta dagli esempi citati la lingua croata è più ricca della lingua italiana in questo settore. Forma con facilità i termini che appartengono alla vita contadina e alla vita degli animali.

2.2.2. Il suffisso -iti forma verbi tratti da sostantivi e indicanti oggetti concreti. I verbi hanno significati diversi e formano vari sottogruppi.

2.2.2.1. Alcuni verbi significano ' 'l' azione fatta con il nome': bradviti (da bradva) asciare 'sgrossare con l'ascia'; jedriti (da jedro) - veleggiare 'navigare a vela'.

Alcuni verbi croati in -iti non hanno suffissati equivalenti italiani, ma soltanto costrutti sintattici costituiti da un verbo transitivo e dal nome (che traduce la base del verbo croato) preceduto dalla preposizione con; oppure dei verbi parasintetici: kundačiti (da kundak) 'battere col calcio (del fucile)'; krečiti (da kreč) 'imbiancare con calce' o il parasintetico incalcinare, formato dal nome calcina mediante il prefisso in- e il suffisso -are.

2.2.2.2. Il suffisso croato -iti forma verbi denominali che significano 'fornire o dare l'oggetto che è la base del verbo' o 'coprire con tale oggetto': biberiti (da biber) pepare 'condire con pepe; gnojiti (da gnoj) - concimare/letamare 'spargere il concime/il letame sul terreno'; hraniti (da hrana) - alimentare 'dare alimento'; soliti (da sol) salare 'trattare un cibo con sale'; šećeriti (da šećer) - zuccherare 'rendere dolce con lo zucchero'.

Molti verbi derivati, formati mediante il suffisso -iti da basi nominali indicanti vari oggetti concreti, non hanno forme equivalenti derivate nella lingua italiana. Sono per lo più i suffissati croati che appartengono al settore dell' agricoltura, dell'agronomia, della zootecnìa e dell'economia in generale. Il croato è molto più ricco dell'italiano in questo settore, e forma con facilità i verbi con questo significato, mentre l'italiano non ha spesso la possibilità di tradurre questi verbi con un verbo formato, derivato o composto; ma dobbiamo ricorrere a forme analitiche, vuol dire a vari costrutti sintattici (verbo seguito dal nome con, o senza, preposizione): krmiti (da krma 'foraggio') 'dare il foraggio al bestiame'; lojiti (da loj) 'ungere di sego'; mastiti (da mast) 'ungere con grasso', mediti (da med) 'addolcire con miele'; pepeliti (da pepeo) 'coprire/imbrattare/spargere di cenere' (o il parasintetico incenerare che è abbastanza raro); zobiti (da zob) 'dare l'avena al bestiame'; žiriti (da žir) 'ingrassare con ghiande'; živičiti (da živica) 'cingere di siepe (viva)'. 
2.2.2.3. Il suffisso croato -iti serve a formare verbi che significano 'fare l'oggetto' che è la base del derivato verbale: gnijezditi se (da gnijezdo) - nidificare 'fare il nido' o il verbo parasintetico annidarsi 'farsi il nido'; poditi (da pod) - pavimentare 'dotare di pavimento o fare il pavimento'; rubiti (da rub) - orlare 'fornire di orlo'; e il verbo tavaniti (da tavan) - soffittare 'munire del soffitto o fare il soffitto'.

Molti di questi verbi non hanno suffissati equivalenti in italiano, ma dobbiamo ricorrere a costrutti analitici o a verbi parasintetici: mostiti (da most) 'gettare un ponte'; plastiti (da plast 'mucchio di fieno') 'ammucchiare il fieno'; snopiti (da snop) 'riunire e legare in covoni o accovonare' o 'legare in fascio o affasciare'; žljebiti (da žljeb) 'fare la grondaia' o 'scanalare'.

Sono termini del settore dell'agricoltura, dell'edilizia e dell'economia in generale.

2.2.2.4. Il suffisso croato -iti forma verbi che significano 'diventare nome/come nome o simile al nome' che è la base del derivato: lediti se (da led) - gelare, gelarsi, ghiacciare, ghiacciarsi; rojiti se (da roj) - sciamare 'formare un nuovo sciame detto delle api'; zrniti se (da zrno) - granire 'fare i chicchi, i granelli, detto del grano o di altri cereali'.

Alcuni di questi suffissati non hanno verbi equivalenti in italiano, formati mediante suffissi, ma soltanto vari costrutti sintattici o verbi parasintetici: jatiti se (da jato) 'adunarsi a stormi'; kameniti se (da kamen) - 'divenire pietra o come pietra e il parasintetico 'impietrire'; prištiti se (da prišt) 'coprirsi di pustole'.

2.2.3. Aggiungiamo ancora alcuni verbi con significati particolari: parničiti (da parnica) - processare; plijeniti (da plijen) - saccheggiare; temeljiti (da temelj) - basare, fig. fondare. ${ }^{1}$

Alcuni di questi verbi croati non hanno forme equivalenti formate mediante suffissi nella lingua italiana, ma solo dei costrutti sintattici o dei verbi parasintetici: okviriti (da okvir) 'incorniciare, intelaiare'; taboriti (da tabor) 'mettere/ far alloggiare in campo o accampare'; trubiti (da truba) 'suonare la tromba o strombettare'; zvoniti (da zvono) 'suonare la campana o scampanare'.

2.2.4. Menzioniamo ancora un gruppo di verbi tratti da basi nominali indicanti concetti diversi: cariniti (da carina) - daziare 'gravare di dazio'; graničiti (da granica) confinare; štetiti (da šteta) - danneggiare 'far danno'.

Alcuni verbi che appartengono a questo gruppo non hanno suffissati verbali equivalenti in italiano, ma solo dei costrutti sintattici che traducono il significato dei verbi croati formati: kulučiti (da kuluk) 'fare lavori tributari o faticosi' (oppure i verbi parasintetici usati in senso figurato: 'sfacchinare, affacchinare'); il verbo sjedničiti (da sjednica) tradotto con 'passare/trascorrere il tempo alle sedute'; e il verbo croato stotiniti se (da stotina) 'moltiplicarsi a centinaia'.

1 L'equivalente formale e semantico del verbo pečatiti, tratto dal nome pečat, è il verbo italiano sigillare. La coscienza linguistica del parlante di oggi sente il legame di questo verbo con la base sigillo. 
2.3. Il suffisso croato -irati serve a formare verbi tratti da basi nominali straniere.

2.3.1. Un gruppo di verbi indica l'azione esercitata dal nome che è la base del verbo: harpunirati (da harpun) - arpionare 'colpire con l'arpione'; telefonirati/telegrafirati (dai nomi telefon e telegraf) - telefonare/telegrafare 'comunicare col telefono/telegrafo'; torpedirati (dal nome torpedo) - silurare 'colpire con siluro; sponzorirati (da sponzor) - sponsorizzare 'finanziare con intento pubblicitario'.

2.3.2. Un gruppo assai numeroso è costituito da verbi che indicano l'azione del verbo transitivo (accompagnato dal nome in funzione di oggetto diretto): akcentirati (da akcent) - accentare 'mettere l'accento'; anketirati (da anketa) - inchiestare 'fare un'inchiesta'; intervjuirati (da intervju) - intervistare 'fare un' intervista. ${ }^{2}$

2.4. Il suffisso -ovati/-evati forma verbi che indicano varie azioni in rapporto al significato della base del verbo: gozbovati (da gozba) - banchettare 'partecipare a banchetti'; putovati (da put) - viaggiare 'fare viaggi'; stupnjevati (da stupanj, stupnja) graduare 'dividere in gradi, ordinare per gradi'. ${ }^{3}$

Alcuni verbi croati non hanno in italiano verbi equivalenti formati mediante suffissi: hladovati (da hlad) 'stare all'ombra, pigliare il fresco'; mirovati (da mir) 'starsene in pace, star quieto e ferno'; školovati (da škola) 'far studiare, mandar alle scuole, mandar agli studi', e l'equivalente del verbo riflessivo školovati se è il suffissato italiano 'studiare'; zavjetovati se (da zavjet) 'far voto di qcs.'.

2.4.1. Quando la base del verbo è un nome indicante persona, il verbo significa 'essere tale persona o vivere, comportarsi, agire come tale persona': agovati (da aga) 'esercitare il potere di agà'; banovati (da ban) 'essere bano'; biskupovati (da biskup) 'essere vescovo'; djevovati (da djeva) 'essere ragazza, vivere da ragazza'; knezovati (da knez) 'essere principe (o conte)'; papovati (da papa) 'essere papa'; pašovati (da paša) 'essere pascià'; popovati (da pop) 'essere prete, esercitare il ministero del prete, fare il prete'; šegrtovati (da šegrt) 'fare l'apprendista'; učiteljevati (da učitelj) 'fare il maestro/l'insegnante, essere maestro/insegnante'.

Come risulta dagli esempi citati il croato sembra più ricco dell'italiano nella formazione di tali verbi; l'italiano impiega vari costrutti sintattici per esprimere il significato di questi suffissati croati.

2.4.2. Alcuni verbi indicano l'azione del verbo transitivo (accompagnato dal sostantivo in funzione di oggetto diretto): filmovati (da film) - filmare 'girare un film'; objedovati (da objed) - pranzare 'consumare il pranzo'. Il croato forma con facilità i verbi con questo significato, ma solo pochi verbi croati hanno verbi equivalenti formati

2 Il verbo croato kuvertirati, tratto dal nome kuverta, non viene incluso in nessun gruppo semantico. Viene tradotto in italiano con il verbo parasintetico imbustare, formato dal sostantivo busta mediante due affissi (il prefisso im- e il suffisso -are) o con il costrutto sintattico 'mettere in una busta'.

3 La coscienza linguistica del parlante di oggi sente il legame di questo verbo con la base grado da cui deriva. 
mediante suffissi nella traduzione italiana. Il verbo croato viene tradotto con diversi costrutti sintattici: božićevati (da Božić) 'festeggiare/passare il Natale'; doručkovati (da doručak, doručka) 'fare colazione'; korjenovati (da korjen) 'estrarre la radice'; pirovati (da pir), svadbovati (da svadba) 'celebrare le nozze'; uskrsovati (da Uskrs) 'festeggiare/passare la Pasqua'.

2.4.3. Se la base del verbo è il nome che indica il tempo, allora il verbo significa 'passare/trascorrere il tempo' indicato dal nome. Di solito gli equivalenti dei verbi croati sono diversi costrutti sintattici: danovati (da dan) 'passare/trascorrere la giornata'; ljetovati (da ljeto) 'passare/trascorrere l'estate'; vjekovati (da vijek) passare i propri giorni'; zimovati (da zima) 'passare/trascorrere l'inverno' o il verbo parasintetico svernare formato dal nome verno (aferesi di inverno) mediante il prefisso s- e il suffisso -are.

\section{I VERBI DEAGGETTIVALI}

I suffissati con base aggettivale si dividono in due grandi gruppi per quanto riguarda il loro significato:

a) il primo gruppo è costituito da verbi che significano 'rendere qcs. conforme al significato dell' aggettivo' che è la base del derivato.

b) il secondo gruppo è costituito da verbi che significano 'diventare aggettivo' che è la base del derivato.

3.1. Il suffisso croato -ati serve a formare verbi con base aggettivale i quali hanno $i$ due significati menzionati.

3.1.1. I verbi del primo gruppo (a) significano 'rendere aggettivo': svjetlati (da svjetao) - lucidare 'rendere lucido', lustrare 'rendere lustro'; veličati (da velik), in senso figurato - magnificare 'rendere magnifico, esaltare con lodi'.

3.1.2. I verbi del secondo gruppo (b) significano 'diventare aggettivo': bređati (da bređa) - diventar incinta, gravida; gorčati (da gorak) - diventar amaro.

3.1.3. Alcuni verbi croati hanno tutti e due i significati, e l'aggettivo che è la base del derivato è al grado comparativo: kraćati (da kraći) 'divenire più corto', 'farsi più corto'; lakšati (da lakši) 'facilitare', ' rendere più facile'; ljepšati (da ljepši) 'rendere più bello, abbellire', 'diventar più bello, abbellirsi'.

L'equivalente italiano è il verbo formato mediante un suffisso (svjetlati - lucidare, lustrare, lakšati - facilitare), il parasintetico (duljati - allungare, allungarsi, ljepšati - abbellire, abbellirsi) e il costrutto sintattico (gorčati - divenir amaro, ljepšati - rendere più bello, divenire più bello) ${ }^{4}$

E' da notare che l'aggettivo (bello) del verbo parasintetico italiano è al grado positivo (a- + bello + -ire), mentre nel costrutto sintattico italiano l'aggettivo è al grado comparativo (rendere/diventare più bello); quindi il costrutto sintattico italiano traduce molto meglio il significatro del verbo croato. 
3.2. I verbi croati formati mediante il suffisso -iti si dividono in più gruppi riguardo al loro significato.

3.2.1. Il verbi del primo gruppo significano 'far diventare aggettivo, rendere aggettivo': bijeliti - imbiancare 'rendere bianco'; bistriti - chiarificare/purificare 'rendere chiaro/ puro/limpido; crniti - annerire 'rendere nero'; rosso'; čistiti - nettare 'rendere netto'; kiseliti - acidificare 'rendere acido'; mlačiti - intiepidire 'rendere tiepido'; pitomiti - addomesticare 'rendere domestico'; prazniti - vuotare 'rendere vuoto'; zeleniti 'colorire o tingere di verde, rendere verde'; žutiti 'colorire o tingere di giallo, rendere giallo, ingiallire.

Come risulta da alcuni degli esempi citati il verbo derivato italiano formato mediante un suffisso è l'equivalente semantico e formale del verbo croato: bistriti - chiarificare/purificare, kiseliti - acidificare; čistiti - nettare, prazniti - vuotare.

L'equivalente semantico e formale del verbo croato è spesso il verbo parasintetico italiano: bijeliti - imbiancare, crniti - annerire, mlačiti - intiepidire, pitomiti - addomesticare, žutiti - ingiallire. Inoltre il verbo croato viene tradotto con un costrutto sintattico analitico che contiene l' aggettivo che traduce la base del derivato croato: zeleniti 'colorire o tingere di verde, rendere verde', žutiti 'colorire o tingere di giallo, rendere giallo'.5

3.2.2. Il suffisso croato -iti forma verbi che significano 'diventare aggettivo o come aggettivo': ćelaviti - incalvire; matoriti - invecchiare; mlohaviti - illanguidire/ infiacchire; slabiti - indebolire; truliti - marcire, ukočiti se - irrigidire (irrigidirsi).

L'equivalente semantico e formale del verbo croato è il suffissato italiano (truliti marcire) o il verbo parasintetico formato dall'aggettivo che traduce la base del verbo croato mediante un prefisso (in-/il-/im-/ir-) e un suffisso (-are/-ire): ćelaviti - incalvire, matoriti - invecchiare, mlohaviti - illanguidire/ infiacchire, slabiti - indebolire, ukočiti se - irrigidire (irrigidirsi).

Inoltre il significato del suffissato croato viene espresso mediante vari costrutti sintattici: ćelaviti 'diventar calvo', matoriti 'diventar vecchio, mlohaviti 'diventar fiacco', slabiti 'diventar debole'.

In croato i verbi riflessivi formati mediante il suffisso -iti possono avere questo significato: bližiti se - avvicinarsi; dlakaviti se 'divenir peloso, coprirsi di peli'; grbaviti se - ingobbire, ingobbirsi 'divenir gobbo'; mladiti se - ringiovanire 'ritornar giovane'; vedriti se - rasserenarsi, 'diventare sereno, ritornare sereno'. ${ }^{6}$

5 Come risulta dagli esempi citati appare chiaro che la base dei verbi è costituita molto spesso dagli aggettivi di colore.

6 Il prefisso ri- appare spesso integrato ad un altro prefisso come in rasserenarsi (a-) e ringiovanire (rin-). Se i verbi si considerano in una prospettiva diacronica si ricollegano con i verbi asserenare (non usato più, in Zingarelli) e ingiovanire (raro, in Zingarelli). La coscienza linguistica dei parlanti di oggi considera questi verbi come dei verbi parasintetici, formati dagli aggettivi sereno e giovane, con doppio prefisso. 
3.2.3. Il suffisso croato -iti serve a formare suffissati che significano 'essere aggettivo o come aggettivo': goropaditi se - smaniare, infuriare 'essere furioso/smanioso'; lakomiti se 'essere avido/ingordo di qcs.'. ${ }^{7}$

Il verbo equivalente italiano è il verbo parasintetico (goropaditi se - infuriare) o il verbo derivato formato mediante un suffisso (goropaditi se - smaniare).

Se non si hanno a disposiszione i mezzi lessicali, si può ricorrere a vari costrutti sintattici (lakomiti se - essere avido/ingordo di qcs.).

3.3. Il suffisso croato -jeti forma i suffissati con base aggettivale i quali indicano 'diventare quello che significa l'aggettivo': hladnjeti - raffreddare 'diventare freddo'; tamnjeti - oscurarsi 'diventare oscuro'.

La base dei verbi croati e dei verbi italiani è costituita molto spesso dagli aggettivi di colore: bijeljeti (da bijel) - imbiancare/imbianchire 'diventare bianco'; crnjeti (da crn) - annerire 'diventare nero'; crvenjeti - diventare rosso; plavjeti (da plav) 'diventare/ritornare azzurro'; rumenjeti (da rumen) 'diventare rosso'; zelenjeti (da zelen) verdeggiare 'diventare verde'; žutjeti (da žut) - ingiallire 'diventare giallo'.

L'equivalente semantico e formale del verbo croato è il verbo derivato italiano, formato mediante un suffisso (zelenjeti - verdeggiare). Il verbo parasintetico italiano è molto spesso l'equivalente formale e semantico del verbo croato: bijeljeti - imbiancare/imbianchire, crnjeti - annerire, hladnjeti - raffreddare, žutjeti - ingiallire.

Il costrutto sintattico, costituito dal verbo diventare e dall'aggettivo che traduce la base del verbo croato, esprime bene il significato del suffissato croato: rumenjeti 'diventare rosso', plavjeti 'diventare/ritornare azzurro', rumenjeti 'diventare rosso'.

3.3.1. Va messo in rilievo che i suffissati croati in -iti e in -jeti, formati dagli aggettivi di colore, possono avere spesso significato uguale e vengono usati con il pronome riflessivo: crnjeti 'crniti se', crvenjeti 'crveniti se', plavjeti 'plaviti se', zelenjeti 'zeleniti se', žutjeti 'žutiti se'. Prevalgono di gran lunga i verbi che indicano i colori.

3.3.2. Va sottolineato che esiste spesso l'opposizione tra verbi in -iti e verbi in -jeti:

bijeliti - imbiancare (v.tr.) 'far diventare/rendere bianco'

bijeljeti - imbiancare/imbianchire (v.intr.) 'diventare bianco'.

Dunque esiste spesso l'opposizione tra verbi in -iti e in -jeti (e verbi in -iti impiegati con il pronome riflessivo) indicanti vari colori:

crniti - annerire (v.tr.) 'rendere/far diventare nero', 'colorire di nero'

crnjeti 'crniti se' - annerire (v.intr. v.intr.pron.) 'diventare nero'

zeleniti - 'rendere/far diventare verde', 'colorire di verde'

zelenjeti 'zeleniti se' - verdeggiare (v.intr.) 'diventare verde' suffissato), anche se il significato dei verbi viene piuttosto collegato dai parlanti di oggi con gli aggettivi furioso e smanioso. 
žutiti - ingiallire (v.tr.) 'rendere/far diventare giallo', 'colorire di giallo'

žutjeti 'žutiti se' - ingiallire (v.intr.) 'diventare giallo'.

Queste opposizioni vengono espresse in italiano con l'uso del verbo transitivo (annerire - il fumo annerisce le pareti; ingiallire - ingiallire i capelli) oppure con l'uso del verbo intransitivo (annerire - le pareti anneriscono; ingiallire - le biade ingialliscono). Viene impiegato il costrutto sintattico 'rendere/far diventare aggettivo' per esprimere il significato del verbo transitivo (rendere/far diventare nero, giallo, verde) o il sintagma 'diventare aggettivo' (diventare nero, giallo, verde) per esprimere il significato del verbo intransitivo.

3.4. Il suffisso croato -ovati forma verbi derivati che possono avere significati diversi, ma prevale il significato 'essere aggettivo': mladovati 'essere giovane'.

L'equivalente semantico e formale di alcuni verbi croati è il verbo italiano derivato, formato mediante un suffisso (-are, -eggiare): ludovati - folleggiare, mudrovati filosofeggiare, vrednovati - valutare.

Ma questi verbi croati vengono tradotti molto spesso con costrutti sintattici. Il croato sembra più ricco dell'italiano nella formazione dei verbi tratti da basi aggettivali, e forma con falicità i verbi in -ovati, i quali vengono tradotti spesso con costrutti sintattici: dobrovati 'essere buono', mladovati 'essere giovane', plahovati 'essere timido', tvrdovati 'essere avaro o spilorcio'.

3.5. Il suffisso croato -izirati forma verbi tratti da basi aggettivali straniere.

Gli equivalenti italiani sono i derivati formati mediante il suffisso -izzare, che forma verbi italiani derivati da basi aggettivali. ${ }^{8}$ Così si hanno i verbi: banalizirati - banalizzare, centralizirati - centralizzare, modernizirati - modernizzare, normalizirati normalizzare, stabilizirati - stabilizzare.

Il significato del verbo croato formato mediante il suffisso -izirati viene tradotto in italiano con i sintagmi verbali ('rendere aggettivo' o 'diventare aggettivo'): banalizirati v.tr. 'rendere banale', v.intr.(pron.) 'diventare banale'.

\section{I VERBI USATI IN SENSO FIGURATO}

I verbi derivati possono essere usati pure in senso figurato e formare delle strutture fraseologiche.

4.1. Il verbo italiano bastonare, derivato dal nome bastone, è l'equivalente semantico e formale del verbo croato batinati. Il verbo può essere impiegato in senso figurato, e allora perde il significato proprio 'percuotere con un bastone', perché la base del verbo (il nome bastone) perde il significato proprio: non è più 'mezzo con cui si per-

8 Per il verbo in -izzare derivato dalla base nominale vedi 2.3.1. - il verbo sponsorizzare, derivato dal nome inglese sponsor. 
cuote/batte qualcuno fisicamente'; quindi il verbo significa 'attaccare con critiche violente': con poche parole l'hanno bastonato.

4.2. Il verbo italiano spronare, derivato dal sostantivo sprone, traduce il verbo croato mamuzati, derivato dal sostantivo mamuza. Il verbo può perdere il significato proprio: 'stimolare il cavallo con gli sproni per incitarlo a correre', e il nome sprone non è più 'oggetto con cui si sprona il cavallo'; quindi il verbo viene usato in senso figurato per stimolare qualcuno in un' attività: spronare qcn. a studiare (allo studio) significa 'stimolare, incitare, indurre qcn. con esortazioni a fare qcs.'.

4.3. Il verbo italiano remare, derivato dal sostantivo remo, è l'equivalente semantico e formale del verbo croato veslati e significa 'maneggiare i remi al fine di imprimere il movimento ad una imbarcazione'. Il verbo può perdere il significato proprio, e allora il sostantivo remo non è più 'oggetto con cui si compie l'azione espressa dal verbo'; e il verbo remare, impiegato con l'avverbio contro, forma la struttura fraseologica remare contro 'ostacolare o rallentare dall'interno l'attività di un gruppo o di una organizzazione'.

4.4. Il verbo italiano filtrare, derivato dal nome filtro, traduce il verbo croato filtrirati. Il verbo può perdere il significato proprio 'passare un liquido, un gas e sim. attraverso un filtro per purificarli' e può essere usato in senso figurato 'elaborare mentalmente': filtrare un'esperienza, filtrare un avvenimento. Nel linguaggio giornalistico viene usato in senso figurato 'riuscire a passare, a trapelare': per quanto segreta, la notizia filtrò ugualmente (vijest je procurila).

4.5. Il verbo italiano silurare, derivato dal nome siluro, è l'equivalente semantico e formale del verbo croato torpedirati 'colpire con siluro'. Se il verbo silurare perde il significato proprio, viene usato in senso figurato e allora può significare 'mandare a monte qcs., far fallire qcs.': silurare una proposta di legge.

\section{CONCLUSIONE}

5.1. In questo articolo vengono esaminati i suffissati verbali tratti da basi nominali e aggettivali. L'approccio è contrastivo, croato - italiano, quindi, vengono confrontati i meccanismi formativi delle due lingue.

5.2. La nostra analisi di suffissati esamina i verbi croati con base nominale e con base aggettivale e cerca gli equivalenti italiani. I suffissati croati presentano una grande diversità di significati. Gli equivalenti italiani esprimono tutti i loro significati; le corrispondenze sono valide dal duplice punto di vista: formale e semantico.

5.3. A un'unità lessicale croata corrisponde spesso più di un'unità lessicale italiana: un suffissato e un parasintetico.

Gli equivalenti semantici e formali dei verbi croati formati da basi nominali e aggettivali mediante suffissi sono: 
5.3.1. I verbi italiani formati mediante suffissi: -are (bastonare, lucidare) -eggiare (danneggiare, verdeggiare), -ificare (nidificare, acidificare), -ire (marcire), -itare (facilitare), -izzare (sponsorizzare, modernizzare).

5.3.2. I parasintetici italiani, tratti da nomi e da aggettivi e formati mediante affissi (prefissi: a-, in-/il-/im-/ir-, ri-, s- e suffissi: -are, -ire): accampare, aggomitolare, illanguidire, imbiancare, imbianchire, ingiallire, irrigidire, rasserenarsi, ringiovanire, svernare.

5.4. A un'unità lessicale croata corrisponde molto spesso un costrutto sintatticosemantico.

Il costrutto sintattico è l'equivalente semantico del verbo derivato croato.

5.4.1. Il costrutto sintattico-semantico è costituito dal verbo e dal nome (con o senza preposizione) che traduce la base del verbo croato, se si tratta di verbi denominali: danovati 'passare/trascorrere la giornata', kundačiti 'battere col calcio del fucile', orgijati 'far baldoria', stotiniti se 'moltiplicarsi a centinaia'.

5.4.2. Il costrutto sintattico-semantico è costituito dal verbo e dall' aggettivo che traduce la base del verbo croato, se si tratta di verbi deaggettivali: dobrovati 'essere buono', mladovati 'essere giovane', duljati 'divenntare più lungo', kraćati 'diventare più corto'.

5.5. Il croato è più ricco dell'italiano in alcuni settori e forma con facilità suffissati denominali e deaggettivali:

5.5.1. Nel sottocodice ecclesiastico: đakoniti 'ordinare qcn. diacono', papovati 'essere papa', popiti 'ordinare qcn. prete', popiti se 'diventare prete', popovati 'essere prete, fare il prete'.

5.5.2. Nel sottocodice della vita contadina e della vita degli animali: jatiti se 'adunarsi a stormi', kulučiti 'fare lavori tributari', janjiti se, koziti se, maciti se, prasiti se, šteniti se, teliti se, ždrijebiti se 'figliare detto di pecora, capra, gatta, scrofa, cagna, mucca'.

5.5.3. Nel settore dell'agricoltura, dell'agronomia, della zootecnia, dell'edilizia e dell' economia in generale: krmiti 'dare il foraggio al bestiame', mediti 'addolcire con miele', mostiti 'gettare un ponte', zobiti 'dare l'avena al bestiame, žiriti 'ingrassare con ghiande', živičiti 'cingere di siepe (viva)', žljebiti 'fare la grondaia'.

5.5.4. Il croato è più ricco dell'italiano nell'esprimere il modo di comportarsi e di agire della persona che svolge una certa attività: agovati 'esercitare il potere di agà', banovati 'essere bano', knezovati 'essere principe/conte', nonché nell'esprimere il modo di passare varie feste: božićevati 'passare/festeggiare il Natale', pirovati/svadbovati 'celebrare/festeggiare le nozze', uskrsovati 'passare/festeggiare la Pasqua'. 


\section{BIBLIOGRAFIA}

Anić,V.(1994) Rječnik hrvatskoga jezika, Novi Liber, Zagreb.

Babić,S.(1991) Tvorba riječi u hrvatskom književnom jeziku. Nacrt za gramatiku, Hrvatska akademija znanosti i umjetnosti, Globus, Zagreb.

Cortelazzo,M.,Cardinale,U.(1988) Dizionario di parole nuove 1964-1987, Torino. Dardano,M.(1978)La formazione delle parole nell'italiano di oggi, Bulzoni, Roma. Deanović,M.-Jernej,J.(1992) Talijansko-hrvatski rječnik, Školska knjiga, Zagreb. Deanović,M.-Jernej,J.(1991) Hrvatsko-talijanski rječnik, Školska knjiga, Zagreb. Forconi,A.(1990) Dizionario delle nuove parole italiane, Milano.

Marchand,H.,1969, The Categorie and Types of Present-Day English Word-Formation, Monaco di B.

Quarantotto,C.(1987) Dizionario del nuovo italiano, Roma.

Tekavčić,P.(1980) Grammatica storica dell'italiano, III: Lessico, il Mulino, Bologna.

Vučetić,Z.(1979) Suvremeni talijanski jezik-Tvorba riječi, Liber, Zagreb.

Vučetić,Z.(1989) Contributo allo studio della suffissazione nominale nell'italiano contemporaneo. Raffronto contrastivo, Linguistica XXIX, Ljubljana, pp.81-99.

Vučetić,Z.(1994) Contributo allo studio della suffissazaione aggettivale nell'italiano contemporaneo, Linguistica XXXIV,2, Ljubljana, pp.49-61.

Zingarelli,N.(1996) Vocabolario della lingua italiana, Dodicesima edizione, Zanichelli, Bologna.

Povzetek

K PREUČEVANJU TVORBE S PRIPONAMI V SODOBNI ITALIJANŠČINI

Prispevek se ukvarja z glagolskimi izpeljankami iz samostalnikov in pridevnikov. Metoda je kontrastivna, in sicer za italijanski in hrvaški glagol. Oblikovno in pomensko so hrvaškim izpeljanim glagolom ustrezniki italijanski bastonare, lucidare, danneggiare, verdeggiare, nidificare, acidificare, marcire, facilitare, sponzorizzare, modernizzare, pogostoma pa tudi parasintetične tvorbe, kot accampare, aggomitolare, imbiancare, imbianchire, infuriare, ingiallire, incalvire, rasserenarsi, ringiovanire. Velikokrat pa hrvaški izpeljani glagol $v$ italijanక̌cini nima ustreznice v izpeljanem glagolu, pač pa v raznih sintagmah, kjer ob glagolu najdemo samostalnik ali pridevnik, tako danovati 'passare/trascorrere la giornata', kundačiti 'battere col calcio del fucile', mladovati 'essere giovane', kraćati 'divenir più corto'.

Iz nabranega gradiva je mogoče trditi, da je hrvaški jezik v nekaterih pojmovnih poljih bogatejši, tako $v$ religiozni terminologiji (papovati, popovati), pa tudi v izrazih iz kmečkega življenja in živalskega sveta (kolučiti, teliti se), iz poljedelstva, pastirstva, gospodarstva (krmiti, mostiti, zobiti, živinčiti). Hrvaščina tudi laže tvori glagole, ki pomenijo obnašanje ali neko dejavnost (agovati, banovati, knezovati, pašovati) ali pa izražajo neko praznovanje (božičevati, pirovati, svadbovati, uskrsovati). 